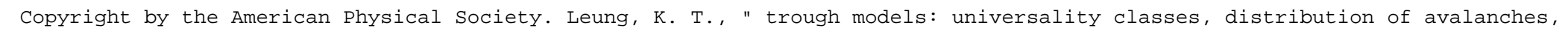
and cluster sizes," Phys. Rev. A 46, 6211 (1992); DOI: http://dx.doi.org/10.1103/PhysRevA.46.6211

PHYSICAL REVIEW A

\title{
Trough models: Universality classes, distribution of avalanches, and cluster sizes
}

\author{
Kwan-tai Leung \\ Department of Physics, Virginia Polytechnic Institute and State University, Blacksburg, Virginia 24061 \\ and Institute of Physics, Academia Sinica, Taipei, Taiwan 11529, Republic of China
}

(Received 13 February 1992)

\begin{abstract}
Extensions of the one-dimensional two-state trough model introduced by Carlson, Chayes, Grannan, and Swindle (CCGS) [Phys. Rev. A 42, 2467 (1990)] are considered. In particular, I investigate what kinds of physical processes are relevant to its scaling behavior. Short-range rearrangements of trough positions (slide events), which were neglected by CCGS, are shown to be irrelevant. By a simple modification of the dynamics, however, I obtain universality classes characterized by a single parameter. For trough models in general, including the two-state and the "limited local" sandpile models, asymptotically exact relations between the distribution of trough-trough distances and that of the mass of avalanches are derived. They yield moment relations in agreement with Krug's [J. Stat. Phys. 66, 1635 (1992)]. All results are verified by simulations.
\end{abstract}

PACS number(s): 05.40. $+\mathrm{j}, 05.60 .+\mathrm{w}, 64.60 . \mathrm{Ht}, 02.50 .+\mathrm{s}$

\section{INTRODUCTION}

Bak, Tang, and Wiesenfeld [1] (BTW) made the remarkable observation that many dissipative dynamical systems in nature exhibiting scale-invariant properties can be generally understood in terms of the notion of "self-organized criticality." While the concept is reminiscent of traditional critical phenomena, it is characterized by threshold instability, with no need of parameter tuning in reaching scale-invariant states.

A pile of sand, modeled by certain cellular automata, is often used as the paradigm of self-organized criticality. First introduced by BTW, and systematically studied with computer simulation by Kadanoff et al. [2], these cellular automation models exhibit nontrivial scaling behavior governed by one kind of threshold instability or another. To elucidate the nature of such behavior, Carlson, Chayes, Grannan, and Swindle [3] (CCGS) identified certain sites, which they called troughs, as those that trap grains sliding down the slope. By definition, the distribution of troughs limits the extent of avalanches. Although the idea was applied specifically to a certain class of onedimensional models, namely the "limited local" model of Kadanoff et al. [2], it seems to be useful in understanding the presence or the lack of scaling in other models as well.

CCGS identified birth, death, slides, and coalescence of troughs as the events triggered by random dropping of a grain on a pile. The trough terminology not only provides an efficient numerical algorithm for simulating sandpile models $[3,4]$, it also offers some hope for a more tractable analytical approach [5]. To illustrate their points, they proposed a simple two-state model and showed that it has a phase transition characterized by the density of troughs $\rho$. Although its criticality requires tuning the birth rate, it has the following important properties: (i) The mean distance between troughs, $1 / \rho$, scales with the system size $L$ as $L^{\theta}$; and (ii) with $0<\theta<1$ at the critical point, there exists nontrivial multiscaling in the distribution of avalanches [6]. These are qualitatively similar to those of sandpile models [2], suggesting a similar physical mechanism is at work.

In connection with the trough model, a few interesting topics deserve further investigation. First, within the simple two-state model, what are the relevant modifications to the rules of dynamics that will yield additional universality classes? This issue is significant because the model is probably the simplest one to display nontrivial multiscaling. Second, aided by its simplicity, what other results can we derive and generalize to more complex sandpile models? Third, what simplifications should be removed and what extra features should be added in order to achieve a closer correspondence to sandpile models? In the following, I will present my attempt in answering these questions.

\section{THE ORIGINAL TWO-STATE MODEL}

CCGS defined the two-state trough model on a onedimensional lattice. A site is either occupied by a trough $(n=1)$ or empty $(n=0)$. The dynamical rules mirror those of sandpiles: A site $i$ is randomly picked (corresponding to random addition of a grain in sandpiles). If $n_{i}=0$, a trough is either born at rate $\lambda$ (birth), or a coalescence is performed at rate unity provided that there are troughs on its left and its right, at sites $i_{L}$ and $i_{R}$. A coalescence amounts to removing the troughs at $i_{L}$ and $i_{R}$ and filling the empty site conjugate to $i$ at $i_{C}=i_{R}+i_{L}-i$. If $n_{i}=1$, then the trough is removed at rate $\delta$ (death).

CCGS showed that a phase transition exists with a critical point at $\lambda=1$ for $\delta>0$. They found that $\rho(L) \sim L^{-\theta}$ with $\theta=1, \frac{1}{2}$, or 0 for $\lambda<1, \lambda=1$, or $\lambda>1$, respectively. They analyzed the case of open boundaries [6], and concluded by simulations that closed boundaries yield the same results.

To facilitate my discussion, I now rederive CCGS's results on finite-size effects associated with different sets of 
boundary conditions, by means of very simple physical arguments. For convenience, I define a cluster to be an interval of zeros bounded either by ones or the boundaries. The left-most cluster will be labeled as $S_{L}$, and the right-most one as $S_{R}$. Only coalescence events will be affected by the boundary conditions: For an open boundary, a coalescence event occurring at the last cluster simply removes the last trough. For a closed boundary, the last trough is removed and the empty site at $i_{C}$ is filled. Notice that there is a net change of -1 or 0 in the total number of troughs $\rho L$ for coalescence near an open or a closed boundary.

For infinite system, there always exist a trough on the left and another on the right of the site $i$ being picked (even if $\rho=0$ ). Thus a coalescence event always contributes a term $-(1-\rho)$ to $d \rho / d t$,

$$
\frac{d \rho}{d t}=\lambda(1-\rho)-\delta \rho-(1-\rho) \text {. }
$$

Now I consider finite-size effects on the basis of this equation. The left and right boundary are located at $i=0$ and $L+1$.

\section{A. Left-closed, right-open boundary}

For a finite system, the density has nontrivial spatial dependence (see Sec. V below). However, this does not complicate our analysis because the "grain" is dropped on a randomly chosen site $i$. What matters to $d \rho / d t$ is the mean density $\rho$, regardless of spatial inhomogeneity. When a coalescence event happens on the cluster $S_{L}$, which is bounded by a wall to its left and a trough to its right, there is no change in $\rho$. This must be excluded from Eq. (1). Denote the mean cluster size by $s=1 / \rho$. The probability of $i$ not belonging to $S_{L}$ is thus $1-s / L$, and the probability of such a site being empty is $1-\rho L /(L-s)$ (I expect $s_{L}$ to scale as the spatial average $s$, as confirmed by simulations). So we have

$$
\frac{d \rho}{d t}=(\lambda-1)(1-\rho)-\delta \rho+\frac{1}{\rho L} \text {. }
$$

Its steady-state solution yields precisely the same results as found by CCGS, with the desirable stability. If the right boundary is also closed (not interesting for sandpile because no outflow of sand is allowed), only the amplitudes of scaling get changed.

\section{B. Both boundaries open, type I}

Here I try to mimic a sandpile with open boundaries by keeping track of a special site near the center designated as the "top" of the pile $(T)$. In a sandpile, the left (or right) nearest neighbor of $T$, by definition of $T$, is always a trough for avalanches occurring on the right (or left) side of $T$. This translates to $n_{T} \equiv 1$ for all time in the two-state model. Finite-size correction to Eq. (1) arises when a coalescence is chosen on either clusters adjacent to $T$, in which case $\rho$ is unchanged. So we have the same results as in Sec. II A.

\section{Both boundaries open, type II}

This is the boundary condition considered by CCGS It differs from Sec. II B in that no special site $T$ is designated. At first glance, there seems to be no simple correction to Eq. (1), since any coalescence event always results in one less trough. Examining closer, however, reveals that the finite-size effects are embedded in the stochasticity of the dynamics. To see this, consider $\lambda=1$. The cancellation between birth and coalescence in the deterministic Eq. (1) actually means that $\rho L$ changes by +1 or -1 due to these two events with equal probability. That implies $\rho(t) L$ is equivalent to the trajectory $x(t)$ of a random walk in one dimension, wandering between two walls at $x=0$ and $L$, with unit steps biased towards $x=0$ by a probability $x \delta / L$. Wandering further away from $x=0$ results in stronger pulling back. From the exact solution of the master equation for such a random walk, the mean distance from $x=0$ is indeed proportional to $\sqrt{L / \delta}$ with a variance proportional to $L$. Thus $\rho(L) \sim 1 / \sqrt{L}$. By properly incorporating the bias between +1 and -1 steps, the above bias random walk turns out to be the most efficient way to check the scaling behavior of $\rho$ for any $\lambda$ and boundary conditions.

\section{VARIATIONS OF THE TWO-STATE MODEL}

\section{A. Irrelevance of slide events}

As noted by CCGS, if the trough on the left at $i_{L}$ during coalescence is formed by the grain dropped at $i=i_{L}+1$, then the number of trough is unchanged. The net effect is a shift to the left by one step for the trough on the right of $i$. CCGS called it a slide event. Another situation where a slide can also occur but with much less frequency is when $i$ is a (shallow) trough, and $i-1$ a stable site. These events occur in sandpiles, but they have not been included in the two-state model.

CCGS suggested that the absence of slide events in the original two-state model may be one of the factors accounting for the differences in scaling behavior from sandpiles. Now I argue that slide events are irrelevant to scaling behavior of the two-state model (but not true for sandpile models [5]). First, notice that slides in the bulk merely result in short-range rearrangements of the spatial distribution of troughs, causing no net change in the number $\rho L$. These rearrangements are irrelevant to $d \rho / d t$ by virtue of the randomness in the position of the grain added. Alternatively, if slides occur at rate $\epsilon$, then they contribute two terms, $\epsilon n_{i+1}\left(1-n_{i}\right)-\epsilon n_{i}\left(1-n_{i-1}\right)$, to $d n_{i} / d t$. By summing over $i$ to get $d \rho / d t$, these extra terms cancel out in pairs.

For finite-size effects, I consider the case with leftclosed and right-open boundary. The only correction comes in when a trough at $i=1$ is picked and a slide is executed (although effectively the same as a death event, it is an independent process). This process gives an extra term $-\epsilon \rho / L$ to Eq. (2), leading to an $O(1 / L)$ correction to $\rho(L)$. Thus the asymptotic scaling behavior is not changed by slides. This expectation is confirmed numerically, for slides accounting for up to $90 \%$ of all possible 


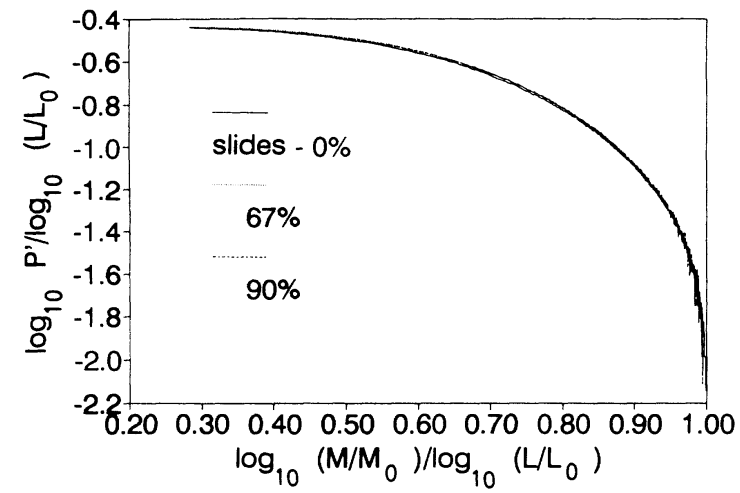

FIG. 1. Multiscaling fits of the mass of bulk avalanches in the two-state model, for slides representing different fractions of all events. This demonstrates its irrelevance. Parameters are $M_{0}=0.085, L_{0}=0.084$. For clarity, only $L=512$ is drawn; curves for different $L$ 's practically overlap on each other.

events. The multiscaling are also checked and no systematic difference from $\epsilon=0$ is detected [7] (see Fig. 1).

\section{B. Other universality classes}

Within the two-state model, there is a very simple modification to the rules of dynamics that results in new
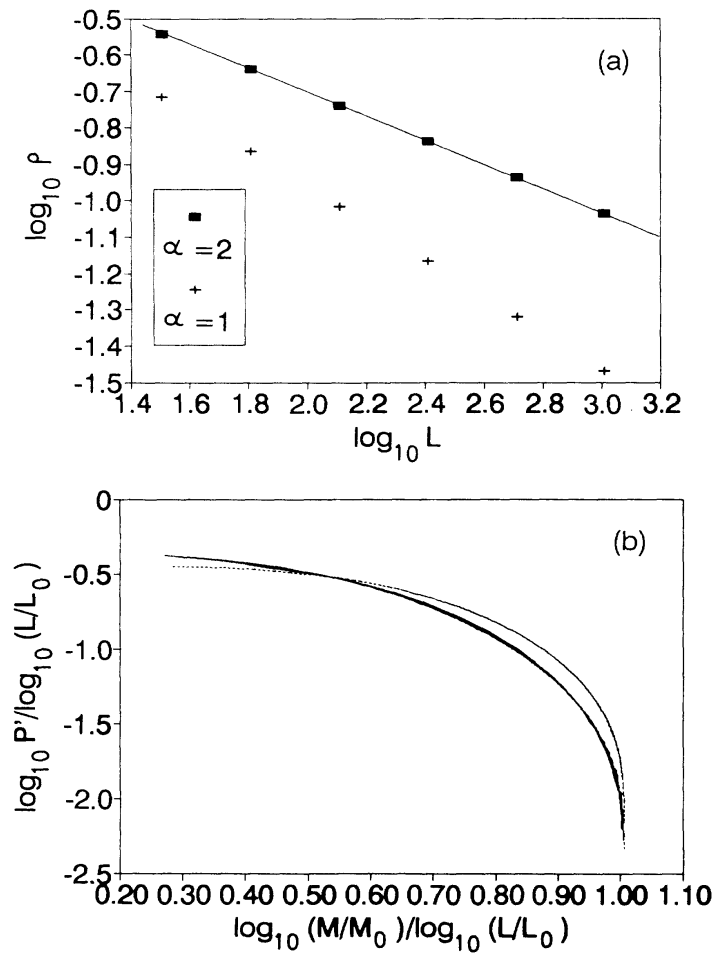

FIG. 2. A modified death rate with $\alpha=2$ gives new critical behavior in (a) the density of troughs: the straight line has slope $-\frac{1}{3}$, while $\alpha=1$ has slope $-\frac{1}{2}$; and (b) multiscaling fit to the distribution of bulk avalanches for $L=32-512$. $M_{0}=0.1$, $L_{0}=0.105$. To demonstrate the differences, the dashed line is for $\alpha=1$ taken from Fig. 1 . universality classes. From Eq. (2), the steady-state condition gives

$$
\delta \rho=\frac{1}{\rho L},
$$

which yields $\rho(L)=1 / \sqrt{\delta L}$. If the term for death events is generalized to $\delta \rho^{\alpha}$, with arbitrary $\alpha$, then we have

$$
\rho(L)=\left(\frac{1}{\delta L}\right)^{1 /(\alpha+1)},
$$

i.e., a one-parameter family of different universality classes. For integral $\alpha$, the death of troughs becomes a multisite process. For example, the case of $\alpha=2$ involves checking a second random site in determining the fate of a trough. This has been checked by simulation. Besides $\rho(L)$, the multiscaling spectra are also different from those of $\alpha=1$ (see Fig. 2).

\section{SOME EXACT RESULTS}

\section{A. Distribution functions}

One of the most appealing features of the two-state model is the appearance of a "mesoscopic" length scale $\langle s\rangle=1 / \rho$. In the thermodynamic limit at criticality, $\langle s\rangle \rightarrow \infty$, but $\langle s\rangle / L \rightarrow 0$. This leads to nontrivial scaling in dynamical quantities such as the sizes of avalanches [6]. In contrast, $\langle s\rangle \sim L$ below criticality $(\lambda<1)$ so that all averages scale trivially with integral powers of $L$, even though avalanches also occur over all possible scales from 1 to $L$. Avalanche in the limited local [2] and the two-state models occurs only during a coalescence event. Its size is given $M=i-i_{L}$ (in units of the number of falling grains per step [2] $N_{F}$ ).

Krug [4] argued that the average avalanche size $\langle M\rangle$ is determined not by the first moment, but by the second moment of the cluster size. The simple reason for the first part of the statement is that an avalanche of a given size $M$ can only be initiated in a cluster of size $s>M$. Thus, the statistical weights of various cluster sizes that enter $\langle M\rangle$ differ from those of $\langle s\rangle$.

Using this fact, it is possible to generalize Krug's results by deriving the full distribution of avalanche $P(M)$ in terms of the cluster size distribution $\widetilde{P}(s)$ : For a given $M$, the number of possible sites to initiate the fall is precisely equal to the number of clusters with sizes $s>M$, so $P(M) \propto \sum_{s=M+1}^{L} \widetilde{P}(s)$. Normalization then gives

$$
P(M)=\frac{\sum_{s=M+1}^{L} \widetilde{P}(s)}{\langle s\rangle-1} .
$$

Hence $\widetilde{P}(s)$ prescribes both the statistics of troughs and of avalanches. This result is exact for the two-state model and is valid for either edge events (with $M N_{F}$ grains of sand falling off the pile) or bulk events (with no falling off). It has been verified numerically up to five digits over the entire range of $M$ for all $L$ probed.

Various moments can then be derived, 


$$
\left\langle M^{n}\right\rangle=\frac{1}{\langle s\rangle-1} \sum_{s=1}^{L} \widetilde{P}(s) \sum_{k=0}^{s-1} k^{n} .
$$

In particular, the first moment agrees asymptotically with Krug's result,

$$
\langle M\rangle=\frac{\left\langle s^{2}\right\rangle-\langle s\rangle}{2[\langle s\rangle-1]},
$$

and the leading term for large $L$ is [8]

$$
\left\langle M^{n}\right\rangle \approx \frac{\left\langle s^{n+1}\right\rangle}{(n+1)\langle s\rangle} .
$$

The relation between $\widetilde{P}(s)$ and $P(M)$ reminds us of the well-known fluctuation-dissipation theorem in equilibrium statistical mechanics [9], since the dynamical quantity $M$ is determined by the fluctuation of the static quantity $s$. Clearly a multiscaling in one implies that for the other. Equations (5)-(8) can be generalized to the $N_{F}=2$ limited local model [2]. In that model, the nontrough sites are either unstable or stable against the addition of a grain. Let $p_{s}$ be the fraction of stable ones among those sites. As a result of the randomness in the position of addition, the parity variable $\sigma_{i} \equiv\left(z_{i} \bmod 2\right)-1$ is random with zero mean [10], where $z_{i}$ is the slope. Since the mean cluster size diverges with $L$, the nontrough sites are asymptotically uncorrelated. This enables us to write for large $L$,

$$
\begin{aligned}
P(M) \propto \delta_{M, 1} & \sum_{s=2}^{L} \widetilde{P}(s)+p_{s} \delta_{M, 1} \sum_{s=3}^{L}(s-2) \widetilde{P}(s) \\
& +\left(1-p_{s}\right)\left[1-\delta_{M, 1}\right] \sum_{s=M+1}^{L} \widetilde{P}(s) \\
= & p_{s} \delta_{M, 1}[\langle s\rangle-1]+\left(1-p_{s}\right) \sum_{s=M+1}^{L} \widetilde{P}(s) .
\end{aligned}
$$

The first term accounts for events when a grain is added to the right nearest neighbor of a trough, the second term for slide events of CCGS, and the third for the rest. The second kind of rare slide events mentioned in Sec. III is not included in $P(M)$. Summing over $M$, one finds the same normalization constant $\langle s\rangle-1$ as for the two-state model. Therefore

$$
P(\boldsymbol{M})=p_{s} \delta_{M, 1}+\left(1-p_{s}\right) \frac{\sum_{s=M+1}^{L} \widetilde{P}(s)}{\langle s\rangle-1}
$$

is the normalized distribution for the $N_{F}=2$ limited local model for large $L$, which has been confirmed with simulations. Up to prefactors involving $p_{s}$ expressions for moments are the same as those for the two-state model. I should emphasize that both $\widetilde{P}(s)$ and $P(M)$ are nontrivial, and the analytic determination of their scaling properties remains an open problem.

\section{B. Relation between edge and bulk cluster sizes}

For the $N_{F}=2$ sandpile, Krug [4] has measured the cluster sizes both in the bulk $\left(s_{b}\right)$ and at the edge $\left(s_{R}\right)$. The two are indeed related: By definition, the bulk average is

$$
\left\langle s_{b}\right\rangle=\frac{\left\langle\sum_{j=1}^{N_{t}} s_{b}^{(j)}\right\rangle}{\left\langle N_{t}\right\rangle},
$$

where $N_{t}$ is the number of troughs. By using a trivial sum rule $\sum_{j} s_{b}^{(j)}=L+1+s_{R}$ and $\rho=N_{t} / L$, we find

$$
\left\langle s_{b}\right\rangle=\frac{L+1-\left\langle s_{R}\right\rangle}{L \rho} .
$$

For $\left\langle s_{R}\right\rangle \sim L^{\theta^{\prime}}$, Eq. (10) offers an interpretation for $\theta^{\prime}$ as an exponent for correction to scaling,

$$
\left\langle s_{b}\right\rangle=A L^{\theta}\left(1-c L^{\theta-1}+\cdots\right) .
$$

This relation applies to any sandpile model with troughs. From this point of view, there seems to be no reason why $\theta^{\prime}$ should be identical to $\theta$, although Krug found that $\theta^{\prime} \approx \theta$ for the sandpile model. For the two-state model, I found $\theta^{\prime} \approx 0.65$, significantly different from $\theta=\frac{1}{2}$.

\section{OTHER RESULTS FROM SIMULATIONS}

The two-state model with and without slide events or modified death rate has been simulated. Since scaling behavior is independent of the death rate, I set $\delta=1$ throughout my simulations. Besides the results described above, I also found nontrivial power-law scaling in both the correlation function $C(i)$ and the trough density profile $\bar{n}(i) \equiv\left\langle n_{i}\right\rangle$. It is expected for a system at criticality $(\lambda=1)$. Figure 3 shows the scaling of $C(i) \equiv\left\langle n_{1} n_{i}\right\rangle-\rho^{2}$ vs $i$. As far as I know, these exponents have not been determined by theory.

By employing $\bar{n}(i) \approx B i^{-\eta}$, and $\rho(L) \sim L^{-\theta}$, it is easy to show that $\bar{n}(i)$ obeys simple finite-size scaling (for $\eta<1$ ),

$$
\bar{n}(i) \approx \rho(L) F(i / L)
$$

with $F(x)=(1-\eta) x^{-\eta}$. Figure 4 displays this scaling behavior. It should be emphasized that this result is not limited to the two-state model; it is valid for any model with power-law dependences in $\bar{n}(i)$ and $\rho(L)$.

Finally, I end with some comments on multiscaling. Customarily, multiscaling of the distribution of a certain

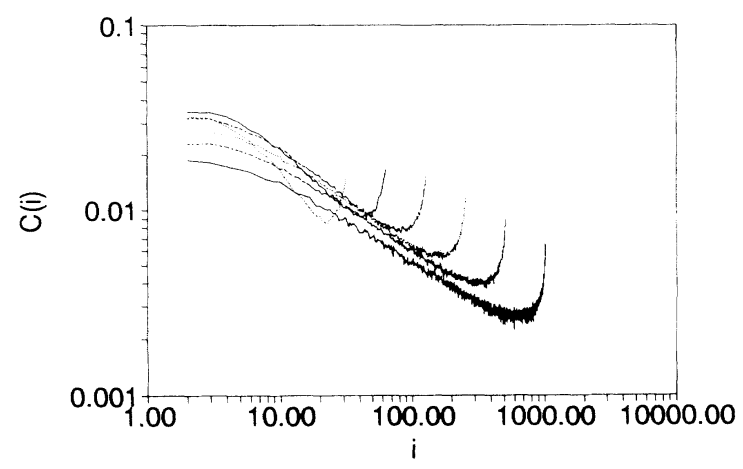

FIG. 3. Power-law decay of trough correlation for $\alpha=1$, $\epsilon=0, L=32-512$, with a slope $\approx-0.47$. 


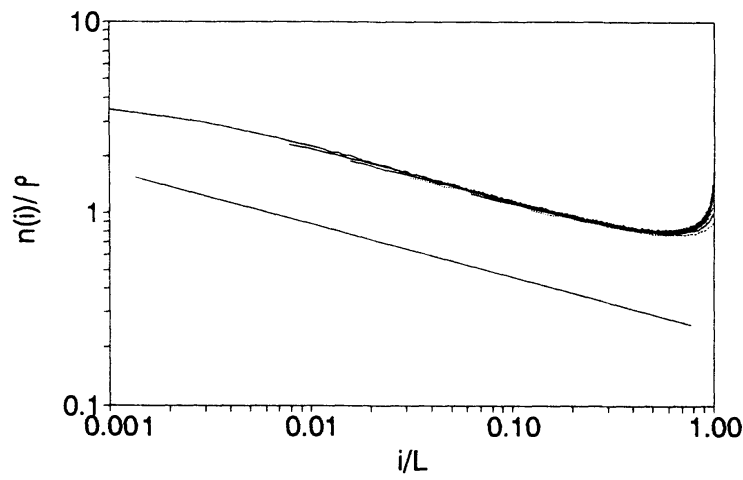

FIG. 4. Finite-size scaling of the local trough density [see Eq. (12)], for $\alpha=1, \epsilon=0, L=32-512$. The slope is -0.28 .

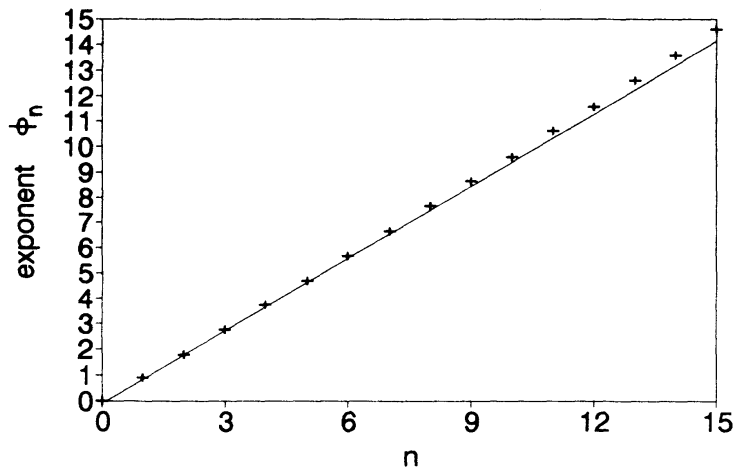

FIG. 5. $\phi_{n}$ is extracted from the moments of edge avalanches $\left\langle M_{e}^{n}\right\rangle \sim L^{\phi_{n}}$, for $\alpha=1, \epsilon=0$. The straight line is for reference. Nonlinearity is barely discernable at large $n$.

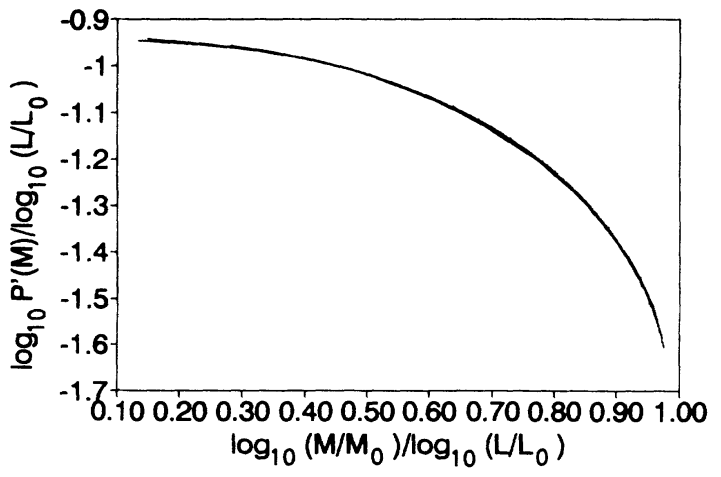

FIG. 6. Multiscaling fit of distribution of edge avalanches for $\alpha=1, \epsilon=0 . M_{0}=0.37, L_{0}=0.31, L=32-512$. quantity $Q$ is explored via two alternative routes: (i) Compute the distribution function and fit it to a multiscaling form [2]; or (ii) extract the exponents of power laws from various moments $\left\langle Q^{n}\right\rangle \sim L^{\phi_{n}}$ and plot $\phi_{n}$ vs $n$ (up to some large $n$ ) [11]. Multiscaling is then inferred by the presence of curvature in the resulting curve. Here I point out that the converse of the second method may fail: The absence of curvature (within the limit of a finite maximum $n$ ) does not imply the absence of multiscaling.

Figure 5 shows such a plot of the exponents for avalanches off the edge. It is as linear as the statistics of data allow. Yet the full distribution unambiguously exhibits multiscaling form (Fig. 6), and the failure of a simple finite-size-scaling fit. In contrast, the distribution of bulk avalanches has a similar multiscaling fit, but the nonlinearity is clearly shown in a plot of exponents. The deficiency of the second method is easily understood by noting that it is limited to a finite set of moments, whereas the full distribution contains information for all moments.

\section{CONCLUSIONS}

The advantage of the two-state model is its simplicity. Most importantly, it presents several diverging intermediate length scales, power-law decays in correlation and density profile (and possibly in temporal decays too), and multiscaling behavior in various distributions. Moreover, some exact results can be derived and easily generalized to sandpile models. Its disadvantage is its restriction to two local states, thus leaving little room for extensions. I showed that it produces critical properties different from sandpiles even when all physical processes of the latter are incorporated. Finally, it requires the tuning of a parameter to get critical.

A limited local model with one nontrough state is one with $N_{F}=1$, which is trivial and has no avalanche. To go beyond the trough model, a generalization to more states is essential. It will then be interesting to prove with the help of the trough representation that the critical point of the sandpile system is naturally attained via its dynamics alone. Progress along this direction is planned to be reported in a forthcoming publication [5].

\section{ACKNOWLEDGMENTS}

I am most grateful to Joachim Krug for many stimulating discussions and comments on the manuscript, for sharing his insights and results with me prior to publication, and informing me of the latest advances in the field. I also thank John Cardy and Jennifer Chayes for useful conversation. This work is supported by a grant from the National Science Foundation through the Division of Material Research. Numerical works were conducted using the Cornell National Supercomputer Facility, a resource of the Cornell Theory Center, which is funded in part by the National Science Foundation, New York State, the IBM Corporation, and members of the Center's Corporate Research Institute. 
[1] P. Bak, C. Tang, and K. Wiesenfeld, Phys. Rev. Lett. 59, 381 (1987); Phys. Rev. A 38, 364 (1988).

[2] L. P. Kadanoff, S. R. Nagel, L. Wu, and S.-m. Zhou, Phys. Rev. A 39, 6524 (1989).

[3] J. M. Carlson, J. T. Chayes, E. R. Grannan, and G. H. Swindle, Phys. Rev. A 42, 2467 (1990); Phys. Rev. Lett. 65, 2547 (1990).

[4] J. Krug, J. Stat. Phys. 66, 1635 (1992).

[5] K.-t. Leung (unpublished).

[6] Although there is no real mass transport in the two-state model, the terms "avalanches," "open," and "closed" boundary can be defined by analogy to the sandpiles.

[7] About normalization: Throughout this paper, $P$ is normalized by $\sum_{x} P(x)=1$. For multiscaling analysis, I use $P^{\prime}$ with a different normalization as close to Kadanoff's as possible [2]. $P^{\prime}$ equals the accumulative distribution divid- ed by the total number of "grains" added, i.e., total number of sweeps times $L$. Thus $P^{\prime}=m P / L$, where $m$ equals the number of the particular events per sweep.

[8] Krug [4] also arrives at this result by means of a different physical argument.

[9] A. Einstein, Ann. Phys. (Leipzig) 17, 549 (1905); H. B. Callen and T. A. Welton, Phys. Rev. 83, 24 (1951); L. Dekker and F. Haake, Phys. Rev. A 11, 2043 (1975).

[10] Reference [4]; L. P. Kadanoff, A. B. Chhabra, A. J. Kolan, M. J. Feigenbaum, and I. Procaccia, Phys. Rev. A 45, 6095 (1992).

[11] See, e.g., N. Jan, P. H. Poole, A. B. Mac Isaac, D. L. Hunter, and S. S. Manna, in Correlations and Connectivity, edited by H. E. Stanley and N. Ostrowsky (Kluwer Academic, Dordrecht, 1990). 